\title{
Endocannabinoid 2-arachidonoylglycerol protects inflammatory insults from sulfur dioxide inhalation via cannabinoid receptors in the brain
}

Short Title: Endogenous 2-AG protects neuroinflammation from $\mathrm{SO}_{2}$ inhalation

Ben Li, Minjun Chen, Lin Guo, Yang Yun, Guangke Li, Nan Sang*

College of Environment and Resource, Shanxi University, Taiyuan, Shanxi 030006, China. E-mail: 727010594@qq.com

Received 04 February 2016

Revised 05 April 2016

Accepted 24 May 2016

\begin{abstract}
Sulfur dioxide $\left(\mathrm{SO}_{2}\right)$ pollution in the atmospheric environment causes brain inflammatory insult and inflammatory-related microvasculature dysfunction. However, there are currently no effective medications targeting the harmful outcomes from chemical inhalation. Endocannabinoids (eCBs) are involved in neuronal protection against inflammation-induced neuronal injury. The 2-Arachidonoylglycerol (2-AG), the most abundant eCBs and a full agonist for cannabinoid receptors ( $\mathrm{CB} 1$ and $\mathrm{CB} 2$ ), is also capable of suppressing proinflammatory stimuli and improving microvasculature dysfunction. Here, we indicated that endogenous 2-AG protected against neuroinflammation in response to $\mathrm{SO}_{2}$ inhalation by inhibiting the activation of microglia and astrocytes and attenuating the overexpression of inflammatory cytokines, including tumor necrosis factor alpha (TNF-a), interleukin (IL)-1 $\beta$, and inducible nitric oxide synthase (iNOS). In addition, endogenous 2-AG prevented cerebral vasculature dysfunction following $\mathrm{SO}_{2}$ inhalation by inhibiting endothelin 1 (ET-1), vascular cell adhesion molecule-1 (VCAM-1) and intercellular adhesion molecule 1 (ICAM-1) expression, elevating endothelial nitric oxide synthase (eNOS) level, and restoring the imbalance between thromboxane A2 (TXA2) and prostaglandin I2 (PGI2). In addition, the action of endogenous 2-AG on the suppression of inflammatory insult and inflammatory-related microvasculature dysfunction appeared to be mainly mediated by CB1 and $\mathrm{CB} 2$ receptors. Our results provided a mechanistic basis for the development of new therapeutic approaches for protecting brain injuries from $\mathrm{SO}_{2}$ inhalation.
\end{abstract}


Keywords: sulfur dioxide; neuroinflammation; microvasculature dysfunction; 2-arachidonoylglycerol; cannabinoid receptors

* Corresponding author. E-mail: sangnan@sxu.edu.cn (Nan Sang)

\section{Introduction}

Sulfur dioxide $\left(\mathrm{SO}_{2}\right)$ is a key factor for air pollution in urban areas, which has been comprehensively studied due to the combustion of coal, power plants, and factories. These facts force us to revisit the adverse health outcomes of $\mathrm{SO}_{2}$ pollution. Epidemiological investigations reveal that $\mathrm{SO}_{2}$ pollution not only increase the risk of many respiratory diseases (Guo et al., 2014; Liu et al., 2014) but is also associated with circulatory system diseases (Amancio and Nascimento, 2012), such as heart disease (Li et al., 2015; Dong et al., 2015), congenital malformation (Farhi et al., 2014) and liver disease (Tong et al., 2015). Increasing evidence suggests that $\mathrm{SO}_{2}$ inhalation is also linked to neurotoxicity and aggravates the risk for hospitalization and mortality of many brain disorders, including ischemic stroke (Amancio and Nascimento, 2014), cerebral hemorrhage (Ye et al., 2009), epilepsy (Cakmak et al., 2010), neurodevelopmental impairment and cognitive deficit (Lin et al., 2014). In the pathophysiology of these neurological diseases, a state of neuroinflammation accompanied by the alteration of the microvasculature function has been shown to be involved (Grammas et al., 2011; Farkas et al., 2000). In particular, our recent results confirm that acute $\mathrm{SO}_{2}$ treatment causes neuroinflammation and microvasculature dysfunction, including the abnormal expression of inflammatory cytokines (inducible nitric oxide synthase (iNOS), cyclooxygenase-2 (COX-2), and intercellular adhesion molecule 1 (ICAM-1) and endothelial dysfunction related factor (endothelin 1 (ET-1) and endothelial nitric oxide synthase (eNOS)), and exacerbates cerebral ischemic responses (Sang et al., 2010; Yun et al., 2010). Specifically, neuroinflammation following chronic $\mathrm{SO}_{2}$ inhalation above the environmental standard has been reported to impair neuronal behavior and repress memory-related kinase activation and the gene expression of glutamate receptors (Yao et al., 2015). Taken together, these findings suggest that $\mathrm{SO}_{2}$ exposure causes brain insults, and inflammation-related responses might be an important molecular modulator during this 
process.

Endocannabinoids (eCBs) have been reported to be involved in a variety of physiological, pharmacological and pathological processes as an endogenous signaling mediator (Marrs et al., 2010; Pertwee et al., 2010) and have the ability to bind to and functionally activate cannabinoid receptors $(\mathrm{CB} 1$ and $\mathrm{CB} 2)$ to exert anti-inflammatory and neuroprotective properties against harmful insults (Mackie, 2006; Chen et al., 2011). As the most abundant eCBs and a full agonist for CB1 and CB2 receptors (Sugiura et al., 2006), 2-arachidonoylglycerol (2-AG) has been shown to play an important role in protecting neurons from inflammatory stimuli (Panikashvili et al., 2001; Kreutz et al., 2007). Subsequent experimental studies demonstrate that indirect elevation of endogenous 2-AG or direct application of 2-AG protected hippocampal neurons from the adverse effects of interleukine-1 beta (IL-1 $\beta$ ) and lipopolysaccharide (LPS), $\beta$-amyloid stimuli and other brain harmful insults by weakening the inflammatory reactions (Chen et al., 2011; Nomura et al., 2011). Brain microvascular dysfunction is an important determinant of the inflammatory response to brain injury. The experimental evidence shows that neuroinflammation participates in brain microvascular dysfunction (Wu et al., 2012). Interestingly, 2-AG also affects other physiological processes, such as vasculature dysfunction and platelet function (Kvasnicka, 2010; Bouchard et al., 2003). 2-AG abolishes the toxicity effect of ET-1-induced cerebral microvascular responses via CB1/2 receptors (Mechoulam and Shohami, 2007). Thus, we hypothesized that endocannabinoid 2-AG may play a protective role against inflammatory insults in the brain and following microvascular dysfunction in response to $\mathrm{SO}_{2}$ inhalation via $\mathrm{CB} 1 / 2$ receptors.

2-AG is mainly produced from diacylglycerol (DAG) by diacylglycerol lipase (DGL) and is hydrolyzed to arachidonic acid (AA) by monoacylglycerol lipase (MAGL) (Nomura et al., 2011). Thus, MAGL inhibition can elevate endogenous 2-AG level. Whereas, JZL184 is a selective inhibitor of MAGL and has been used for disrupting MAGL and improving the endogenous 2-AG content. In the present study, we established sub-acute $\mathrm{SO}_{2}$ inhalation models $\left(14 \mathrm{mg} / \mathrm{m}^{3}, 6 \mathrm{hr} /\right.$ day, 7 days $)$ in the absence or presence of pre-intraperitoneal injection of JZL184 and investigated the protection of 2-AG against brain neuroinflammation and proinflammatory cytokines-mediated microvascular dysfunction following $\mathrm{SO}_{2}$ inhalation. 


\section{Material and methods}

\subsection{Experimental animals and exposure treatments}

C57BL6 mice, weighing 18-20 g, were purchased from the Center of Experimental Animal of Hebei Province and used according to the guidelines approved by the Institutional Animal Care and Use Committee of Shanxi University. The mice were divided randomly into 5 equal groups, in which each group consist of 8 animals, including vehicle, $\mathrm{SO}_{2}, \mathrm{JZL} 184$ (a monoacylglycerol lipase inhibitor, Cayman, USA) $+\mathrm{SO}_{2}$, SR-1 (SR141716, a CB1 antagonist, Cayman, USA) + JZL184+SO 2, SR-2 (SR144528, a CB2 antagonist, Cayman, USA) + JZL184 $+\mathrm{SO}_{2}$ groups. Similarly, vehicle and $\mathrm{SO}_{2}$ groups were intraperitoneally injected with normal saline, and other groups were intraperitoneally injected with JZL184 (12 mg/kg), SR-1 $(5 \mathrm{mg} / \mathrm{kg})+\mathrm{JZL184}, \mathrm{SR}-2(5 \mathrm{mg} / \mathrm{kg})+\mathrm{JZL} 184$ for 7 days, respectively. All reagents were injected once every two days for a total of three times in a 7-day timespan. Mice were then treated with $\mathrm{SO}_{2}\left(14 \mathrm{mg} / \mathrm{m}^{3}\right)$ for $6 \mathrm{hr} /$ day $(10: 00$ a.m. to 16:00 p.m.) for 7 days. To obtain the desired concentration and generate $\mathrm{SO}_{2}$ gas for distribution among the entire chamber, the $\mathrm{SO}_{2}$ exposure appliance was prepared as previously described (Yao et al., 2015). In addition, the concentration of $\mathrm{SO}_{2}$ exposure in the chamber was monitored by applying a real-time $\mathrm{SO}_{2}$ monitor (FIX660, Wandi, China). An alkali absorption device was used to absorb the gas waste. During the $\mathrm{SO}_{2}$ exposure process, the mice were allowed free movement under normal circumstances, except for access to food and water. The 1-hr China's Ambient Air Quality Standard Grade II of $\mathrm{SO}_{2}$ is $0.5 \mathrm{mg} / \mathrm{m}^{3}$ (GB 3095-2012, 2012). The concentration used in this study was $14 \mathrm{mg} / \mathrm{m}^{3}$ (6 hr/day), then the 24-hr average concentration every day was 3.5 $\mathrm{mg} / \mathrm{m}^{3}$ and it is approximately 7 times higher than the standard. Although the experiments may be viewed as beyond the normal atmosphere encountered in the human environment, two important points must be taken into account. First, animals were subjected to regular periods of extended exposure, with relief periods between protocols $(6 \mathrm{hr} /$ day, for 7 days, with $18 \mathrm{hr}$ between exposures). This finding may provide a corollary to individuals exposed to the gas in an occupational setting. Second, real-time and mean concentrations in the upper and lower airways were higher in humans when compared with rat using a comparative simulation of $\mathrm{SO}_{2}$ gas transport in airway models (Tsujino et al., 2005).

The mice were sacrificed $12 \mathrm{hr}$ after the last exposure, and the cortex was rapidly excised. 
A small piece of tissue was removed and fixed with 4\% paraformaldehyde for immunofluorescent staining, and the remaining tissue was frozen in liquid nitrogen and stored at $-80^{\circ} \mathrm{C}$ in an ultra-cold storage freezer for immunoblot analysis and enzyme-linked immunosorbent assay.

\subsection{Protein isolation and immunoblot analysis}

Proteins were extracted from cortical tissues in ice-cold lysis buffer as previously described (Yao et al., 2015). The lysates were centrifuged at $13,000 \mathrm{r} / \mathrm{min}$ for $15 \mathrm{~min}$ at $4^{\circ} \mathrm{C}$, and protein concentrations were determined using the bicinchoninic acid assay. Briefly, $50 \mu \mathrm{g}$ of total proteins were separated using Sodium dodecyl sulfate (SDS)-polyacrylamide gel electrophoresis (PAGE) and transferred onto a $0.45-\mu \mathrm{m}$ aperture nitrocellulose membrane, and the protein bands were cut and blocked with 3\% bovine serum albumin (BSA) for $1 \mathrm{hr}$. After blocking, the membrane was incubated with rabbit polyclonal primary antibodies at $4{ }^{\circ} \mathrm{C}$ overnight, including polyclonal antibodies ET-1, iNOS, eNOS, VCAM-1, ICAM-1 (volume ratio, 1:200, Bioss, China) and rabbit monoclonal antibodies $\beta$-actin $(1: 1000$, CST, USA). The blot was washed with $0.1 \mathrm{~mol} / \mathrm{L}$ phosphate buffer (PBS) and incubated with a fluorescently labeled secondary antibody (1:5000) (IRDye $800 \mathrm{CW}$ goat anti-rabbit $\operatorname{IgG}(\mathrm{H}+\mathrm{L})$ at room temperature for $1 \mathrm{hr}$, and the blot was scanned and detected using the LI-COR Odyssey (Odyssey sa, Gene, USA) Infrared Fluorescent System.

\subsection{Immunofluorescent staining}

The fixed cortical tissues were embedded in paraffin, cut into 4-6 $\mu \mathrm{m}$ slices, and adhered onto glass slides. The sections were heated for $20 \mathrm{~min}$ at $60^{\circ} \mathrm{C}$ prior to deparaffinization with xylene, and antigen retrieval was performed using citric acid buffer ( $\mathrm{pH}$ 6.0). Next, the sections were blocked with 3\% BSA solution for $30 \mathrm{~min}$ at room temperature and incubated with anti-CD11b/c antibody (1:500, Abcam, USA) and anti-glial fibrillary acidic protein (GFAP) antibody (1:2000, Abcam, USA), a marker of microglia and astrocyte, respectively, overnight at $4^{\circ} \mathrm{C}$; the sections were washed three times with PBS and incubated with anti-rabbit secondary antibodies for $1 \mathrm{hr}$ at room temperature. After staining with 4', 6-diamidino-2-phenylindole (DAPI), a fluorescent stain which binds strongly to DNA, the 
sections were rapidly mounted, coverslipped, and stored in darkness at $4^{\circ} \mathrm{C}$ until images were obtained. The images were then obtained by applying a fluorescent inverted microscope (IX73, Olympus, Japan), in which the relative fluorescence intensity was calculated using Image-pro Express 6.0 software (Media Cybernetics, USA).

\subsection{Measurement of 6-keto-PGF1 $\alpha$ and TXB2 levels}

Because both PGI2 and TXA2 have a very short half-life and are ready to convert to 6-keto-PGF1 $\alpha$ and thromboxane B2 (TXB2), respectively, we measured 6-keto-PGF1 $\alpha$ and TXB2 levels. Briefly, cortical tissues were homogenized in a buffer containing $1 \mathrm{mmol} / \mathrm{L}$ ethylene diamine tetraacetic acid (EDTA), $10 \mathrm{~mol} / \mathrm{L}$ indomethacin, and $0.1 \mathrm{~mol} / \mathrm{L} \mathrm{PBS}$. Homogenates were centrifuged at $12000 \times g$ for $15 \mathrm{~min}$ at $4^{\circ} \mathrm{C}$. Supernatants were removed and assayed in triplicate using a 6-keto-PGF1 $\alpha$ and TXB2 enzyme-linked immunosorbent assay (ELISA) kits according to the manufacturer's guidelines (Cayman, USA).

\subsection{Liquid chromatography-tandem mass spectrometry}

The 2-AG were quantified in the brain tissues of $\mathrm{C} 57 \mathrm{BL} / 6 \mathrm{~J}$ mice using high-performance liquid chromatography coupled with tandem mass spectrometry (LC-MS/MS). The quantification of 2-AG was based on an external standard curve diluted with methanol. All the standards of the analytes were provided by Cayman Chemical (Ann Arbor, MI, USA). The brain tissue was weighed and extracted individually. Frozen samples were placed in extraction solvent containing $2 \mathrm{~mL}$ of acetonitrile in a borosilicate glass tube. After rapid homogenization, the sample was centrifuged at $1500 \times g$ for $10 \mathrm{~min}$. The supernatant was collected and evaporated to dryness under a gentle stream of nitrogen. Then, $200 \mu \mathrm{L}$ of methanol were added to the extract, which was dried under $\mathrm{N}_{2}$ gas. Subsequently, the lipid extract was resuspended using $20 \mu \mathrm{L}$ of methanol, and $5 \mu \mathrm{L}$ were injected for LC-MS/MS analysis. A Waters UPLC system coupled with a triple quadrupole Quattro Premiere/XE mass spectrometer (Q-tof 2, Waters, USA) was used for the analysis. Liquid chromatography was performed using a Waters XSelect CSH C18 column $2.1 \times 75 \mathrm{~mm}, 2.5$ $\mu \mathrm{m}$ (Waters, USA), with $0.1 \%$ formic acid (A) and 100\% acetonitrile (B) as a mobile phase. A flow rate of $400 \mu \mathrm{L} / \mathrm{min}$ was used for gradient elution, which started at $50 \% \mathrm{~B}$, linearly 
increased to $90 \%$ at $2 \mathrm{~min}$ and was subsequently reequilibrated at $50 \%$ B for a further 2 min after a 4-min hold. The column temperature was $45^{\circ} \mathrm{C}$. The samples were kept at $4^{\circ} \mathrm{C}$ in the autosampler until analysis. The targeted LC-MS/MS analysis was performed in multiple reactions monitoring mode (MRM) using the following transitions: the electrospray ionization $(\mathrm{ESI})+\mathrm{MRM}$ transition for $2-\mathrm{AG}$ was $379.4>287.3$. The amounts of $2-\mathrm{AG}$ were calculated from the ratios of peak areas of compounds to external standards and by comparing the results with standard curves.

\subsection{Measurement of TNF- $\alpha$, IL-1 $\beta$}

The enzyme-linked immunosorbent assay (ELISA) kit (Xitang, China) was selected to evaluate the concentration of TNF- $\alpha$ and IL-1 $\beta$ secreted from mouse cortex. Briefly, 0.3-0.5 g of tissue was homogenized in $1 \mathrm{~mL}$ of homogenization buffer $(0.1 \mathrm{~mol} / \mathrm{L}$ phosphate, $\mathrm{pH} 7.4)$, and the lysates were centrifuged at $1000 \times g$ for $20 \mathrm{~min}$. The supernatants were collected and assayed in triplicate according to the protocol of the ELISA kit.

\subsection{Data analysis}

Data are expressed as the mean \pm S.E. and unless stated otherwise, Student's $t$-test and ANOVA with Fisher's least significant difference (LSD) test were used to test for statistical comparison when appropriate, using Origin8.0 Software. Differences were considered significant when $p<0.05$.

\section{Results}

\subsection{MAGL disruption alters endogenous 2-AG level following $\mathrm{SO}_{2}$ inhalation}

To provide direct evidence for the alteration of endogenous 2-AG levels following MAGL inhibition by JZL184, we first detected the 2-AG contents in the cortex under different treatment conditions. These results showed that the chemical inhalation did not alter the endogenous 2-AG content. However, MAGL inhibition by JZL184 pre-treatment significantly elevated the levels of endogenous 2-AG (Fig. 1). This finding suggested that MAGL inhibition by JZL184 increased the content of endogenous 2-AG, which might exert protective effects on neuroinflammation and its related injuries in response to $\mathrm{SO}_{2}$ inhalation. 


\subsection{Endogenous 2-AG decreases astroglia activation and neuroinflammation from $\mathrm{SO}_{2}$}

\section{inhalation}

For brain inflammation, a major status is astroglia cell activation, and the activation astroglia cells release a broad array of chemokines, cytokines, and toxic factors (Sofroniew and Vinters, 2010). To determine whether endogenous 2-AG protects against neuroinflammation from $\mathrm{SO}_{2}$ inhalation, we determined the reactive astrocytes and microglia using specific markers in mouse cortex. These results indicated that $\mathrm{SO}_{2}$ inhalation caused the activation of astrocytes and microglia, and JZL184 subsequently suppressed the elevation (Fig. 2). To confirm the preliminary result, we further detected the release of inflammatory cytokines. As shown in Fig. 3, $\mathrm{SO}_{2}$ exposure increased the expression of iNOS, TNF- $\alpha$ and IL-1 $\beta$, and JZL184 statistically inhibited the overexpression.

\subsection{Endogenous 2-AG protects against inflammatory insults from $\mathrm{SO}_{2}$ inhalation via}

\section{$\mathrm{CB} 1 / 2$ receptors}

eCBs have been reported to protect against inflammation via binding to and functionally activating cannabinoid receptors. To clarify whether endogenous 2-AG protected inflammatory insults from $\mathrm{SO}_{2}$ inhalation via $\mathrm{CB} 1 / 2$ receptors, we treated mice with $\mathrm{SO}_{2}(14$ $\mathrm{mg} / \mathrm{m}^{3}$ ) in the presence of JZL184, SR-1+JZL184, and SR-2+JZL184, and determined the release of inflammatory cytokines, including iNOS, TNF- $\alpha$ and IL-1 $\beta$. As presented in Fig. 3, both SR1 (CB1 antagonist) and SR2 (CB2 antagonist) reversed JZL184-induced suppression of iNOS overproduction in response to $\mathrm{SO}_{2}$ exposure but not TNF- $\alpha$ and IL-1 $\beta$. These results suggested that 2-AG protected against brain inflammation from $\mathrm{SO}_{2}$ inhalation, and the action was partly modulated by $\mathrm{CB} 1 / 2$ receptors.

\subsection{Endogenous 2-AG inhibits abnormality of neuroinflammation-related} microvasculature factors from $\mathrm{SO}_{2}$ inhalation

Following brain inflammation, neuroinflammation-related microvasculature insults are also associated with many neurological diseases (Amancio and Nascimento, 2014; Ye et al., 2009). During this process, the abnormal metabolite of AA, thromboxane A2 (TXA2) and 
prostaglandin I2 (PGI2), which maintains the state of contraction and relaxation of the microvasculature under normal physiological conditions, occurred (Miller, 2006). Here, we first examined the TXA2 and PGI2 expression following $\mathrm{SO}_{2}$ inhalation in the absence or presence of JZL184 pre-treatment. As shown in Fig. 4, $\mathrm{SO}_{2}$ exposure down-regulated PGI2 levels, and JZL184 reversed the change in PGI2. TXA2 demonstrated no significant changes in the absence or presence of JZL184. Coupled with the imbalance between TXA2 and PGI2, the biomarkers of inflammation-related microvasculature dysfunctions, including ET-1, eNOS, ICAM-1, and VCAM-1, caused vascular homeostasis and perturbed vasculature function (Frijns and Kappelle, 2002; Leung et al., 2009). Thus, we further detected the expression of ET-1, eNOS, ICAM-1 and VCAM-1. As shown in Fig. 5, $\mathrm{SO}_{2}$ exposure increased the expression of ET-1, ICAM-1, VCAM-1 and down-regulated the level of eNOS, and JZL184 reversed these alterations. These results suggested that endogenous 2-AG prevents neuroinflammation-related microvasculature dysfunction in response to $\mathrm{SO}_{2}$ inhalation.

\subsection{Endogenous 2-AG reverses neuroinflammation-related microvasculature dysfunction from $\mathrm{SO}_{2}$ inhalation via $\mathrm{CB} 1 / 2$ receptors}

To confirm that endogenous 2-AG protected against inflammation-related microvasculature insults from $\mathrm{SO}_{2}$ inhalation via $\mathrm{CB} 1 / 2$ receptors, we treated mice with $\mathrm{SO}_{2}$ in the presence of JZL184, SR-1+JZL184, and SR-2+JZL184 and further detected the expression of ET-1, eNOS ICAM-1 and VCAM-1. As shown in Fig. 5, both SR-1 and SR-2 abolished endogenous 2-AG-generated suppression of ET-1, ICAM-1 and VCAM-1 following $\mathrm{SO}_{2}$ exposure. However, SR-1, but not SR-2, inhibited the endogenous 2-AG-produced restoration of eNOS downregulation. This finding implicated that elevation of endogenous 2-AG by MAGL inhibition protected against neuroinflammation-related injuries from $\mathrm{SO}_{2}$ inhalation via $\mathrm{CB} 1 / 2$ receptors.

\section{Discussion}

$\mathrm{SO}_{2}$ is a widespread pro-inflammatory stimulus to the central nervous system (CNS) and has been largely studied as a risk factor for neurological disease (Shah et al., 2015; Weisskopf et al., 2015; Amancio and Nascimento, 2014; Yun et al., 2013). Although the accurate 
mechanisms underlying neurological disease induced by $\mathrm{SO}_{2}$ exposure are not completely understood, recent studies indicate that oxidative stress, glial activation, neuroinflammation, and the following microvascular dysfunction may be attributed to the primary pathways (sang et al., 2010; yun et al., 2010; Block and Calderón-Garcidueñas, 2009). Moreover, inflammation in the vasculature has been shown to be involved in vasculature dysfunction-related diseases, such as ischemic stroke (Amancio and Nascimento, 2014) and hypertension (Cero et al., 2016). Recent studies have shown that chronic systemic inflammation causes vasculature dysfunction by down-regulating eNOS expression, and up-regulating ICAM-1 expression in blood vessels occurred in the rostral ventrolateral medulla (RVLM) (Wu et al., 2012). Inflammation in the brain, produced by proinflammatory cytokine interleukin (IL)-1 $\beta$, accelerates brain microvascular leakage and brain edema (Chakraborty et al., 2010). Thus, the control of inflammation and subsequent vasculature dysfunction are an important target to prevent and treat neurological disorders due to $\mathrm{SO}_{2}$ inhalation. In recent years, the pleiotropic effects of endogenous 2-AG occurred for the prevention and treatment of inflammation, and vasculature dysfunction began to be investigated and concerned (Zogopoulos et al., 2013; Grabner et al., 2016). Thus, we proposed that strengthening endogenous 2-AG might represent a potential therapeutic target for the treatment of brain injury from $\mathrm{SO}_{2}$ exposure.

It is well known that microglia are the most important immune defense in the CNS, and they have been reported to produce a variety of pro-inflammatory cytokines, such as tumor necrosis factor (TNF), nitric oxide, interleukin and other neurotoxic substances in response to environmental toxins (Block and Hong, 2005). Although the experimental study showed that astrocytes are not as effective as microglia in pro-inflammation function (Lindsay, 1994), it can be obviously activated and produce a variety of neuronal survival-related factors in response to inflammation stimulates or brain injuries (Aloisi, 1999; Tacconi, 1998). Thus, we first confirmed that $\mathrm{SO}_{2}$ exposure enhanced the activation of astroglia and the expression of iNOS, TNF- $\alpha$ and IL-1 $\beta$, and the processes were observably inhibited by endogenous 2-AG. These results indicated that endogenous 2-AG pose the ability to protect against neuroinflammation from $\mathrm{SO}_{2}$ inhalation.

Vasoactive mediators produced by inflammatory cells may trigger acute cerebrovascular 
diseases. TXA2/PGI2 and ET-1/eNOS are two groups of important vasoactive substances. PGI2 and eNOS exert vasodilatation effects that counterbalance the harmful vasoconstriction effects of TXA2 and ET-1, respectively (Miller, 2006). Microvasculature dysfunction has increasingly been implicated as a crucial player in the pathogenesis of a wide variety of neuroinflammation-induced diseases upon exposure to air pollutants (Grammas et al., 2002). The clinical and animal experiments demonstrated that the two groups of substances are involved in a variety of cerebrovascular diseases (Kunos et al., 2000). Acute and chronic exposures to vehicular source pollutants have also been associated with vasculature dysfunction by altering brain ET-1 and eNOS gene expression (Thomson et al., 2007). Chronic exposure to cigarette smoke causes an imbalance of PGI2 and TXA2 production (Sherratt et al., 1988). Moreover, there are many inflammatory adhesion molecules that contribute to vascular endothelial functional properties, including ICAM-1, VCAM-1 (Frijns and Kappelle, 2002). Thus, we examined the expression of TXA2, PGI2, ET-1, eNOS, ICAM-1, and VCAM-1 and found that $\mathrm{SO}_{2}$ inhalation caused microvasculature dysfunction by elevating ET-1, ICAM-1, and VCAM-1 expression, down-regulating eNOS levels and disrupting the balance between TXA2 and PGI2. However, these inflammation-related microvasculature insults were obviously reversed from $\mathrm{SO}_{2}$ inhalation by elevating endogenous 2-AG via MAGL inhibition, suggesting that endogenous 2-AG contributed to restoration of inflammation-related microvasculature dysfunction caused by $\mathrm{SO}_{2}$ exposure.

eCBs have been reported to exert anti-inflammation and protect microvascular function via the activation of their cannabinoid receptors (Chen et al., 2011; Eljaschewitsch et al., 2006; Mechoulam and Shohami, 2007). In the present study, endogenous 2-AG resulting in downregulation of INOS, ET-1, ICAM-1 and VCAM-1 in response to $\mathrm{SO}_{2}$ inhalation was reversed by both SR-1 and SR-2 (antagonist of CB1 and CB2), while a change in eNOS was only targeted by SR-1. Interestingly, iNOS, but not TNF- $\alpha$ and IL-1 $\beta$, inhibition was significantly restored in the presence of SR-1 and SR-2. These findings implicated that the CB1/2-dependent signaling pathway was the primary mechanism mediating the protective effects of 2-AG from inflammation and inflammation-related microvasculature function, but other mechanisms could not be excluded. Recent evidence suggest that eCBs bind to peroxisome proliferator-activated receptors (PPARs) or vanilloid receptors to exert the effect 
of anti-inflammatory and neuroprotection (Paterniti et al, 2013; Pini et al., 2012; Rockwell et al, 2006), suggesting that other receptors mediate endogenous 2-AG protection against brain inflammatory insult and inflammation-related microvasculature dysfunction from $\mathrm{SO}_{2}$ inhalation.

\section{Conclusions}

In summary, endogenous 2-AG prevented neuroinflammation and its related microvasculature dysfunction from $\mathrm{SO}_{2}$ inhalation, and the action was mediated mainly via CB1/2 receptors. This study provides a mechanistic basis for the development of new therapeutic approaches for protecting against brain inflammation from $\mathrm{SO}_{2}$ inhalation.

\section{Acknowledgments}

This work was supported by the National Science Foundation of China (Nos. 91543203, 21477070, 21377076, 21307079), and the Research Project Supported by Shanxi Scholarship Council of China (No. 2015-006).

\section{References}

Aloisi, F., 1999. The role of microglia and astrocytes in CNS immune surveillance and immunopathology. Adv. Exp. Med. Biol. 468, 123-133.

Amancio, C.T., Nascimento, L.F., 2014. Environmental pollution and deaths due to stroke in a city with low levels of air pollution: ecological time series study. Sao Paulo Med. J. $132(6), 353-358$.

Amancio, C.T., Nascimento, L.F., 2012. Association of sulfur dioxide exposure with circulatory system deaths in a medium-sized city in Brazil. Braz. J. Med. Biol. 45 (11), 1080-1085.

Block, M.1., Hong, J.S., 2005. Microglia and inflammation-mediated neurodegeneration: multiple triggers with a common mechanism. Prog. Neurobiol. 76 (2), 77-98.

Block, M.L., Calderón-Garcidueñas, L., 2009. Air pollution: mechanisms of neuroinflammation and CNS disease. Trends Neurosci. 32 (9), 506-516.

Bouchard, J.F., Lépicier, P., Lamontagne, D., 2003. Contribution of endocannabinoids in the 
vasculature protection afforded by ischemic preconditioning in the isolated rat heart. Life Sci. 72 (16), 1859-1870.

Cakmak, S., Dales, RE., Vidal, C.B., 2010. Air pollution and hospitalization for epilepsy in Chile. Environ. Int. 36 (6), 501-505.

Cero, F.T., Hillestad, V., Sjaastad, I., Yndestad, A., Aukrust, P., Ranheim, T., et al., 2015. Absence of the inflammasome adaptor ASC reduces hypoxia-induced pulmonary hypertension in mice. Am. J. Physiol. Lung Cell. Mol. Physiol. 309 (4), L378-L387.

Chakraborty, S., Kaushik, D.K., Gupta, M., Basu, A., 2010. Inflammasome signaling at the heart of central nervous system pathology. J. Neurosci. Res. 88, 1615- 1631.

Chen, X., Zhang, J., and Chen, C., 2011. Endocannabinoid 2-arachidonoylglycerol protects neurons against b-amyloid insults. Neurosci. 178, 159-168.

Dong, G.H., Wang, J., Zeng, X.W., Chen, L., Qin, X.D., Zhou, Y., et al., 2015. Interactions between air pollution and obesity on blood pressure and hypertension in chinese children. Epidemiology 26 (5), 740-747.

Eljaschewitsch, E., Witting, A., Mawrin, C., Lee, T., Schmidt, P.M., Wolf, S., et al., 2006. The endocannabinoid anandamide protects neurons during CNS inflammation by induction of MKP-1 in microglial cells. Neuron 49, 67-79.

Farhi, A., Boyko, V., Almagor, J., Benenson, I., Segre E., Rudich, Y., et al., 2014. The possible association between exposure to air pollution and the risk for congenital malformations. Environ. Res. 135, 173-180.

Farkas, E., De Jong, G.I., de Vos, R.A., Jansen Steur, E.N., Luiten, P.G., 2000. Pathological features of cerebral cortical capillaries are doubled in Alzheimer's disease and Parkinson's disease. Acta Neuropathol. 100 (4), 395-402.

Frijns, C.J, Kappelle, L.J., 2002. Inflammatory cell adhesion molecules in ischemic cerebrovascular disease. Stroke 33, 2115-2122.

GB 3095-2012, 2012. Ambient Air Quality Standard (In China). Available at: http://www.es.org.cn/download/2012/1-6/2273-1.pdf.

Grabner, G.F., Eichmann, T.O., Wagner, B., Gao, Y., Farzi, A., Taschler, U., et al., 2016. Deletion of monoglyceride lipase in astrocytes attenuates lipopolysaccharide-induced neuroinflammation. J. Biol. Chem. 291 (2), 913-923. 
Grammas, P., Yamada, M., Zlokovic, B., 2002. The cerebromicrovasculature: a key player in the pathogenesis of Alzheimer's disease. J. Alzheimers Dis. 4 (3), 217-223.

Grammas, P., Martinez, J., Miller, B., 2011. Cerebral microvascular endothelium and the pathogenesis of neurodegenerative diseases. Expert Rev. Mo. Med. 13, e19.

Guo, L.J., Zhao, A., Chen, R.J., Kan, H.D., Kuang, X.Y., 2014. Association between ambient air pollution and outpatient visits for acute bronchitis in a Chinese city. Biomed. Environ. Sci. 27 (11), 833-840.

Kunos, G., Járai, Z., Bátkai, S., Goparaju, S.K., Ishac, E.J., Liu, J., et al., 2000. Endocannabinoids as cardiovascular modulators. Chem. Phys. Lipids 108 (1-2), 159-168.

Kreutz, S., Koch, M., Ghadban, C., Korf, H.W., Dehghani, F., 2007. Cannabinoids and neuronal damage: differential effects of THC, AEA and 2-AG on activated microglial cells and degenerating neurons in excitotoxically lesioned rat organotypic hippocampal slice cultures. Exp. Neurol. 203, 246-257.

Kvasnicka, T., 2010. The endocannabinoid system and interference with thrombogenesis. Vnitr. Lek. 56 (2), 127-129.

Leung, J.W., Chung, S.S., Chung, S.K., 2009. Vasculature endothelin-1 over-expression using receptor tyrosine kinase tie-1 promoter leads to more severevascular permeability and blood brain barrier breakdown after transient middle cerebral artery occlusion. Brain Res. $1266,121-129$.

Li, H., Chen, R., Meng, X., Zhao, Z., Cai, J., Wang, C., et al., 2015. Short-term exposure to ambient air pollution and coronary heart disease mortality in 8 Chinese cities. Int. J. Cardiol. 197, 265-270.

Lin, C.C., Yang, S.K., Lin, K.C., Ho, W.C., Hsieh, W.S., Shu, B.C., et al., 2014. Multilevel analysis of air pollution and early childhood neurobehavioral development. Int. J. Environ. Res. Public Health 11 (7), 6827-6841.

Lindsay, R.M., 1994. Neurotrophic growth factors and neurodegenerative diseases: therapeutic potential of the neurotrophins and ciliary neurotrophic factor. Neurobiol. Aging 15, 249-251.

Liu, F., Zhao, Y., Liu, Y.Q., Liu, Y., Sun, J., Huang, M.M., et al., 2014. Asthma and asthma related symptoms in 23,326 Chinese children in relation to indoor and outdoor 
environmental factors: the Seven Northeastern Cities (SNEC) Study. Sci. Total Environ. 497-498, 10-17.

Marrs, W.R., Blankman, J.L., Horne, E.A., Thomazeau, A., Lin, Y.H., Coy, J., et al., 2010. The serine hydrolase ABHD6 controls the accumulation and efficacy of 2-AG at cannabinoid receptors. Nat. Neurosci. 13 (8), 951-957.

Mackie, K., 2006. Cannabinoid receptors as therapeutic targets. Annu. Rev. Pharmacol. Toxicol. 46, 101-122.

Mechoulam, R., Shohami, E., 2007. Endocannabinoids and traumatic brain injury. Mol. Neurobiol. 36 (1), 68-74.

Miller, S.B., 2006. Prostaglandins in health and disease: an overview. Semin. Arthritis Rheum. $36,37-49$.

Nomura, D.K., Morrison, B.E., Blankman, J.L., Long, J.Z., Kinsey, S.G., Marcondes, M. C., et al., 2011. Endocannabinoid hydrolysis generates brain prostaglandins that promote neuroinflammation. Science 334, 809-813.

Panikashvili, D., Simeonidou, C., Ben-Shabat, S., Hanuš, L., Breuer, A., Mechoulam, R., et al., 2001. An endogenous cannabinoid (2-AG) is neuroprotective after brain injury. Nature 413, 527-531.

Paterniti, I., Impellizzeri, D., Crupi, R., Morabito, R., Campolo, M., Esposito, E., et al., 2013. Molecular evidence for the involvement of PPAR- $\delta$ and PPAR- $\gamma$ in anti-inflammatory and neuroprotective activities of palmitoylethanolamide after spinal cord trauma. J. Neuroinflamm. 10, 20.

Pini, A., Mannaioni, G., Pellegrini-Giampietro, D., Passani, M.B., Mastroianni, R., Bani, D., et al., 2012. The role of cannabinoids in inflammatory modulation of allergic respiratory disorders, inflammatory pain and ischemic stroke. Curr. Drug Targets 13 (7), 984-993.

Pertwee, R.G., Howlett, A.C., Abood, M.E., Alexander, S.P., Di Marzo, V., Elphick, M.R., et al., 2010. International Union of Basic and Clinical Pharmacology. LXXIX. Cannabinoid receptors and their ligands: beyond $\mathrm{CB}_{1}$ and $\mathrm{CB}_{2}$. Pharmacol. 62 (4), 588-631.

Rockwell, C.E., Snider, N.T., Thompson, J.T., Vanden Heuvel, J.P., and Kaminski, N.E., 2006. Interleukin-2 suppression by 2-arachidonyl glycerol is mediated through peroxisome proliferator-activated receptor gamma independently of cannabinoid 
receptors 1 and 2. Mol. Pharmacol. 70, 101-111.

Sang, N., Yun, Y., Li, H., Hou, L., Han, M., Li, G.K., 2010. $\mathrm{SO}_{2}$ inhalation contributes to the development and progression of ischemic stroke in the brain. Toxicol. Sci. 114, 226-236.

Sofroniew, M.V., Vinters, H.V., 2010. Astrocytes: biology and pathology. Acta Neuropathol. $119,7-35$.

Sugiura, T., Kishimoto, S., Oka, S., Gokoh, M., 2006. Biochemistry, pharmacology and physiology of 2-arachidonoylglycerol, an endogenous cannabinoid receptor ligand. Prog. Lipid Res. 45 (5), 405-446.

Shah, A.S., Lee, K.K., McAllister, D.A., Hunter, A., Nair, H., Whiteley, W., et al., 2015. Short term exposure to air pollution and stroke: systematic review and meta-analysis. Br. Med. J. 350, h1295.

Sherratt, A.J., Culpepper, B.T., Lubawy, W.C., 1988. Relative participation of the gas phase and total particulate matter in the imbalance in prostacyclin and thromboxane formation seen following chronic cigarette smoke exposure. Prostaglandins Leukot. Essent. Fatty Acids 34 (1), 15-18.

Tacconi, M.T., 1998. Neuronal death: is there a role for astrocytes? Neurochem. Res. 23, $759-765$.

Thomson, E.M., Kumarathasan, P., Calderón-Garcidueñas, L., Vincent, R., 2007. Air pollution alters brain and pituitary endothelin-1 and inducible nitric oxide synthase gene expression. Environ. Res. 105 (2), 224-233.

Tong, L., Li, K., Zhou, Q., 2015. The association between air pollutants and morbidity for diabetes and liver diseases modified by sexes, ages, and seasons in Tianjin, China. Environ. Sci. Pollut. Res. Int. 22 (2), 1215-1219.

Tsujino, I., Kawakami, Y., and Kaneko, A. (2005). Comparative simulation of gas transport in airway models of rat, dog, and human. Inhal. Toxicol. 17, 475-485.

Weisskopf, M.G., Kioumourtzoglou, M.A., Roberts, A.L., 2015. Air pollution and autism spectrum disorders: causal or confounded? Curr. Environ. Health Rep. 2 (4), 430-439.

Wu, K.L., Chan, S.H., Chan, J.Y., 2012. Neuroinflammation and oxidative stress in rostral ventrolateral medulla contribute to neurogenic hypertensioninduced by systemic inflammation. J. Neuroinflammation 9, 212. 
Yao, G., Yue, H., Yun, Y., Sang, N., 2015. Chronic $\mathrm{SO}_{2}$ inhalation above environmental standard impairs neuronal behavior and represses glutamate receptor gene expression and memory-related kinase activation via neuroinflammation in rats. Environ. Res. 137, $85-93$

Ye, Y., Li, X.Y., Chen, K., Liu, Q.M., Xiang, H.Q., 2009. A case-crossover study on the relationship between air pollution and acute onset of cerebral hemorrhage in Hangzhou city. Zhong hua Liu Xing Bing Xue Za Zhi. 30 (8), 816-819.

Yun, Y., Hou, L., Li, G. K., and Sang, N., 2010. $\mathrm{SO}_{2}$ inhalation modulates the expression of apoptosis- related genes in rat hippocampus via its derivatives in vivo. Inhal. Toxicol. 22, 919-929.

Yun, Y., Yao, G., Yue, H., Guo, L., Qin, G., Li, G., et al., 2013. SO $\mathrm{SO}_{2}$ inhalation causes synaptic injury in rat hippocampus via its derivatives in vivo. Chemosphere 93 (10), 2426-2432.

Zogopoulos, P., Vasileiou, I., Patsouris, E., Theocharis, S., 2013. The neuroprotective role of endocannabinoids against chemical-induced injury and other adverse effects. J. Appl. Toxicol. 33 (4), 246-264. 


\section{Figure legend}

Fig. 1 JZL184 improves the 2-AG level in mice brain after $\mathrm{SO}_{2}$ exposure. The level of 2-AG in the brain of mice under different treatment conditions $(n=8) .{ }^{a} p<0.05$ compared with vehicle controls.

Fig. 2 Endogenous 2-AG suppresses $\mathrm{SO}_{2}$ inhalation caused activtion of microglia and astrocytes in mice brain. (A1) Activated microglial cells (CD11b/c, a microglial marker, red) in the brain of mice in vehicle control, $\mathrm{SO}_{2}$ exposure and $\mathrm{JZL} 184+\mathrm{SO}_{2}$ groups; (A2) Quantifications of CD11b/c positive staining cells under different conditions; (B1) Activated astrocytes (GFAP, an astrocytic marker, red) in the brain of mice in vehicle control, $\mathrm{SO}_{2}$ exposure and JZL184+SO $\mathrm{SO}_{2}$ groups; (B2) Quantifications of GFAP positive staining cells under different conditions. ${ }^{\text {aa }} p<0.01$ compared with vehicle controls; ${ }^{\mathrm{b}} p<0.05$ and ${ }^{\mathrm{bb}} p<0.01$ compared with $\mathrm{SO}_{2}$ groups. Data are averaged from 3 to 4 sections in three groups of mice, $n=8$ mice per group.

Fig. 3 Endogenous 2-AG inhibits neuroinflammation following $\mathrm{SO}_{2}$ inhalation through $\mathrm{CB} 1$ and CB2 receptors. (A) Quantifications of TNF- $\alpha$ contents in cortex of mice exposed to $\mathrm{SO}_{2}$ in the absent or present of JZL184, SR-1+JZL184 and SR-2+JZL184 (n=8); Quantifications of IL-1 $\beta$ contents in cortex of mice exposed to $\mathrm{SO}_{2}$ in the absent or present of JZL184, SR-1+JZL184 and SR-2+JZL184 ( $n=8)$; (C1) Immunoblot analysis of iNOS in cortex of mice exposed to $\mathrm{SO}_{2}$ in the absent or present of JZL184, SR-1+JZL184 and SR-2+JZL184; (C2) Quantifications of iNOS expression under different conditions $(n=8)$. +: with; -: without. ${ }^{\text {aa }} p<0.01$ and ${ }^{\text {aaa }} p<0.001$ compared with vehicle controls; ${ }^{\text {b }} p<0.05$ and ${ }^{\text {bb }} p$ $<0.01$ compared with $\mathrm{SO}_{2}$ group; ${ }^{c} p<0.05$ compared with $\mathrm{SO}_{2}$ group in the presence of JZL184.

Fig. 4 Endogenous 2-AG reverses PGI2 down-regulation in response to $\mathrm{SO}_{2}$ inhalation. Levels of TXA2 (A), PGI2 (B) andTXA2/PGI2 ratio (C) in the cortex of mice under different conditions. ${ }^{\mathrm{a}} p<0.01$ compared with vehicle controls; ${ }^{\mathrm{b}} p<0.05$ and ${ }^{\mathrm{bb}} p<0.01$ compared with 
$\mathrm{SO}_{2}$. Data are averaged from 3 to 4 sections in three groups of mice, $n=8$ mice per group.

Fig. 5 Endogenous 2-AG abolishes ET-1, VCAM-1 and ICAM-1 elevation and eNOS attenuation by $\mathrm{CB} 1 / 2$ receptors. (A1) Immunoblot analysis of ET-1 in cortex of mice exposed to $\mathrm{SO}_{2}$ in the absent or present of JZL184, SR-1+JZL184 and SR-2+JZL184; (A2) Quantifications of ET-1 expression under different conditions ( $n=8)$; (B1) Immunoblot analysis of eNOS in cortex of mice exposed to $\mathrm{SO}_{2}$ in the absent or present of JZL184, SR-1+JZL184 and SR-2+JZL184. (B2) Quantifications of eNOS expression under different conditions $(n=8)$. (C1) Immunoblot analysis of ICAM-1 in the cortex of mice exposed to $\mathrm{SO}_{2}$ in the absent or present of JZL184, SR-1+JZL184 and SR-2+JZL184. (C2) Quantifications of ICAM-1 expression under different conditions $(n=8)$. (D1) Immunoblot analysis of VCAM-1 in the cortex of mice exposed to $\mathrm{SO}_{2}$ in the absent or present of JZL184, SR-1+JZL184 and SR-2+JZL184. (D2) Quantifications of VCAM-1 expression under different conditions $(n=8)$. Abbreviations: +, with; -, without. ${ }^{\mathrm{aa}} p<0.01$ compared with vehicle controls; ${ }^{\mathrm{b}} p<0.05$ compared with $\mathrm{SO}_{2}$ group; ${ }^{\mathrm{c}} p<0.05$ compared with $\mathrm{SO}_{2}$ group in the presence of JZL184. 


\section{JZL184}

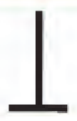

2-AG

MAGL

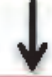

CB1/2

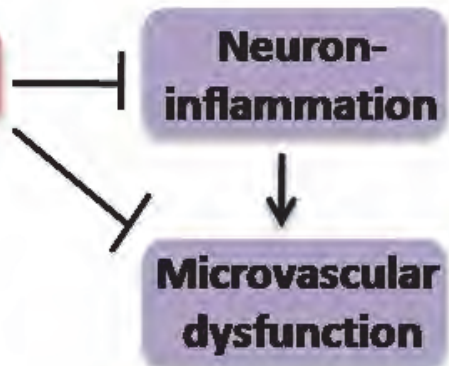

$\mathrm{SO}_{2}$ inhalation 


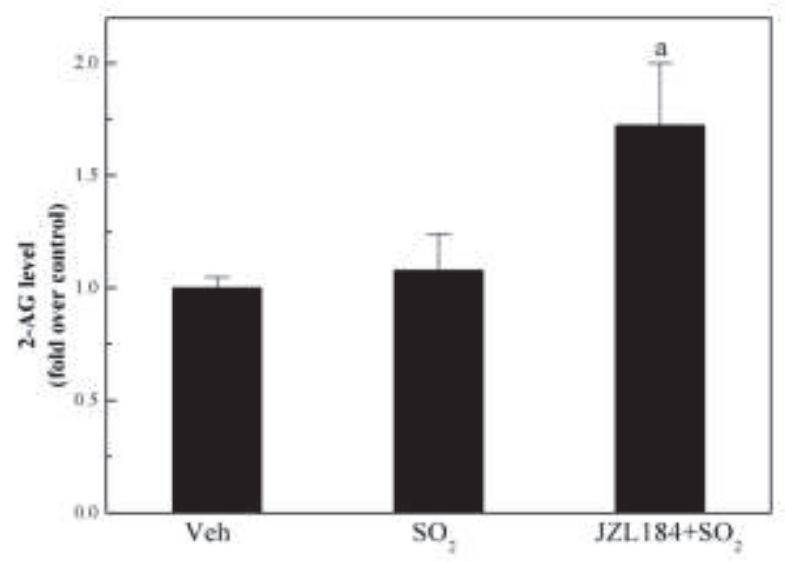

Fig. 1 JZL184 improves the 2-AG level in mice brain after $\mathrm{SO}_{2}$ exposure. The level of $2-\mathrm{AG}$ in the brain of mice under different treatment conditions $(n=8) .{ }^{\mathrm{a}} p<0.05$ compared with vehicle controls.
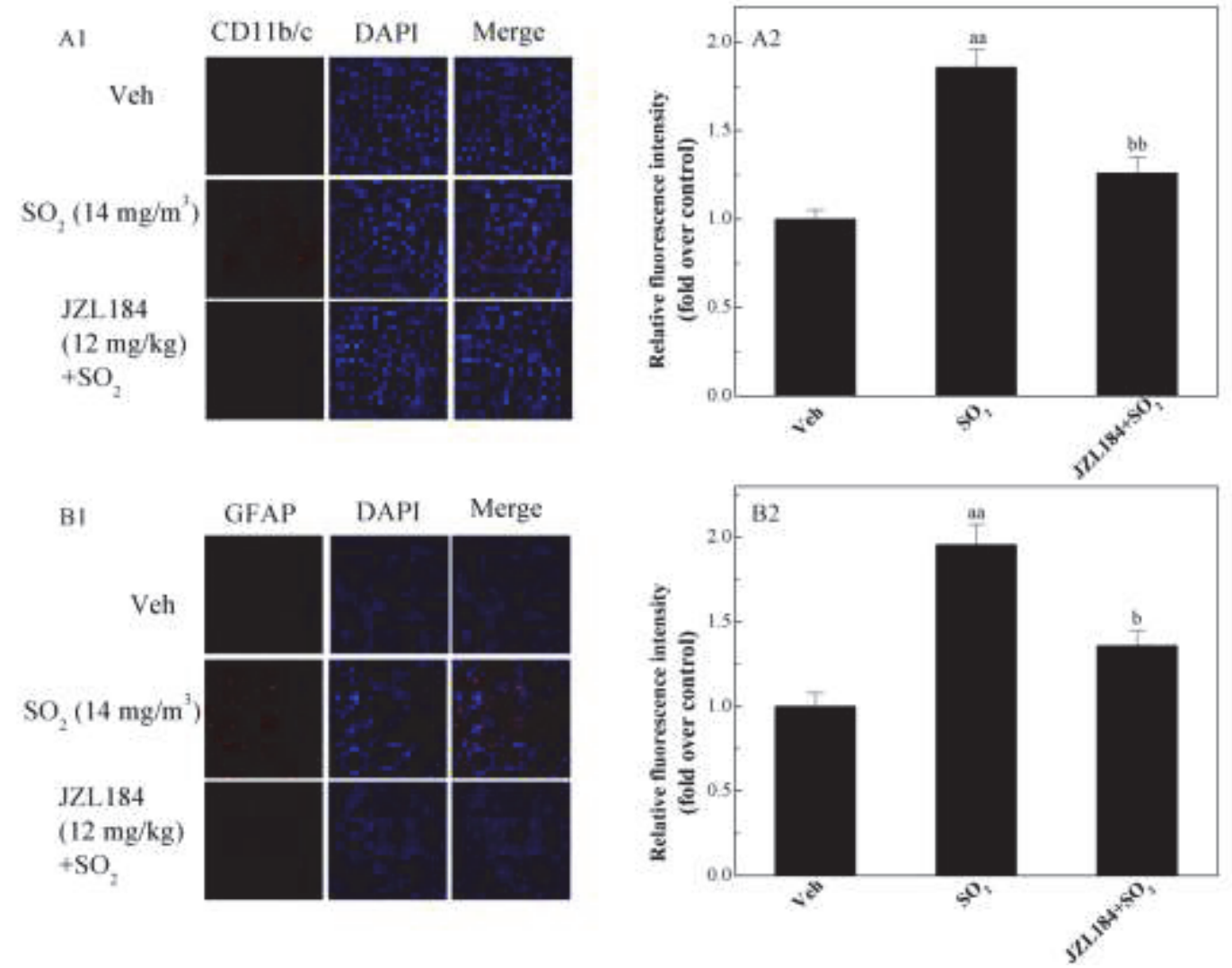

Fig. 2 Endogenous 2-AG suppresses $\mathrm{SO}_{2}$ inhalation caused activtion of microglia and 
astrocytes in mice brain. (A1) Activated microglial cells (CD11b/c, a microglial marker, red) in the brain of mice in vehicle control, $\mathrm{SO}_{2}$ exposure and JZL184+ $\mathrm{SO}_{2}$ groups; (A2) Quantifications of CD11b/c positive staining cells under different conditions; (B1) Activated astrocytes (GFAP, an astrocytic marker, red) in the brain of mice in vehicle control, $\mathrm{SO}_{2}$ exposure and JZL184+SO 2 groups; (B2) Quantifications of GFAP positive staining cells under different conditions. ${ }^{\mathrm{aa}} p<0.01$ compared with vehicle controls; ${ }^{\mathrm{b}} p$ $<0.05$ and ${ }^{\mathrm{bb}} p<0.01$ compared with $\mathrm{SO}_{2}$ groups. Data are averaged from 3 to 4 sections in three groups of mice, $n=8$ mice per group.
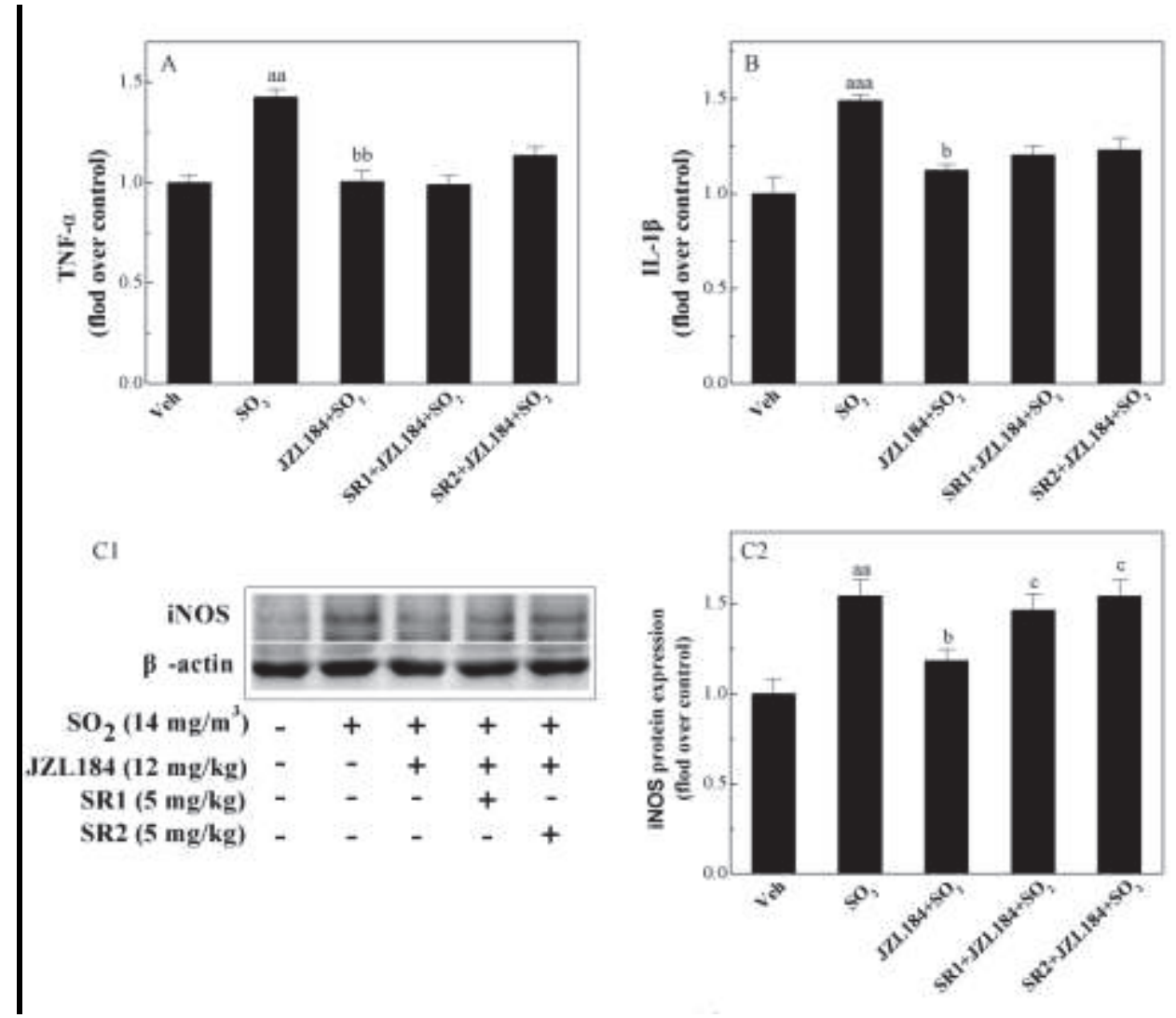

Fig. 3 Endogenous 2-AG inhibits neuroinflammation following $\mathrm{SO}_{2}$ inhalation through CB1 and CB2 receptors. (A) Quantifications of TNF- $\alpha$ contents in cortex of mice exposed to $\mathrm{SO}_{2}$ in the absent or present of JZL184, SR-1+JZL184 and SR-2+JZL184 $(n=8)$; (B) Quantifications of IL-1 $\beta$ contents in cortex of mice exposed to $\mathrm{SO}_{2}$ in the absent or present of JZL184, SR-1+JZL184 and SR-2+JZL184 (n=8); (C1) Immunoblot analysis of iNOS in cortex of mice exposed to $\mathrm{SO}_{2}$ in the absent or present of JZL184, SR-1+JZL184 and SR-2+JZL184; (C2) Quantifications of iNOS expression 
under different conditions ( $n=8)$. +: with; -: without. ${ }^{\text {aa }} p<0.01$ and ${ }^{\text {aaa }} p<0.001$ compared with vehicle controls; ${ }^{\mathrm{b}} p<0.05$ and ${ }^{\mathrm{bb}} p<0.01$ compared with $\mathrm{SO}_{2}$ group; ${ }^{\mathrm{c}} p$ $<0.05$ compared with $\mathrm{SO}_{2}$ group in the presence of JZL184.

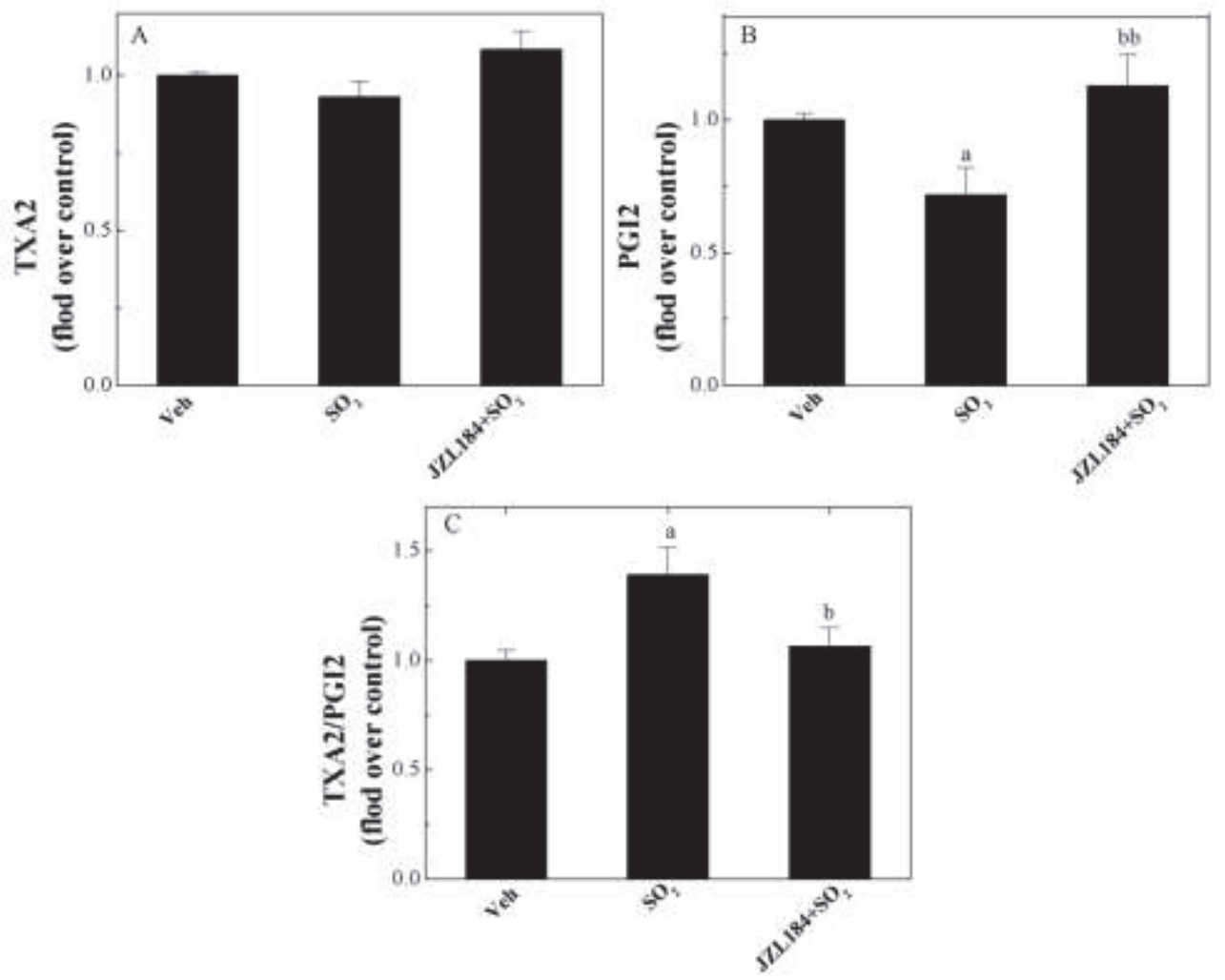

Fig. 4 Endogenous 2-AG reverses PGI2 down-regulation in response to $\mathrm{SO}_{2}$ inhalation. Levels of TXA2 (A), PGI2 (B) andTXA2/PGI2 ratio (C) in the cortex of mice under different conditions. ${ }^{\mathrm{a}} p<0.01$ compared with vehicle controls; ${ }^{\mathrm{b}} p<0.05$ and ${ }^{\mathrm{bb}} p<0.01$ compared with $\mathrm{SO}_{2}$. Data are averaged from 3 to 4 sections in three groups of mice, $n=8$ mice per group. 
A1

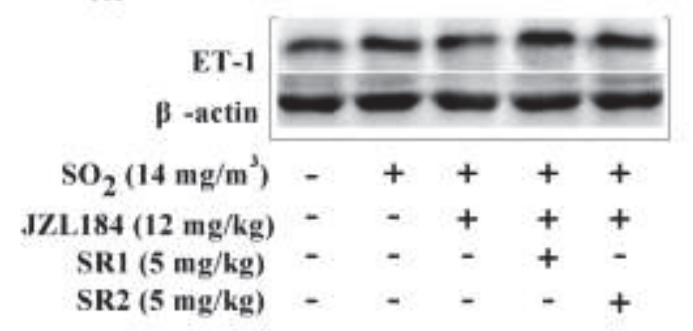

B1

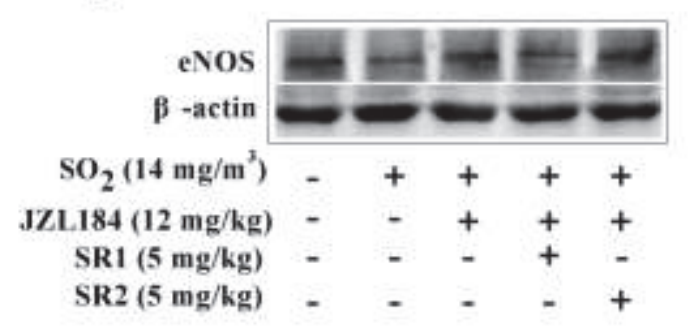

$\mathrm{Cl}$

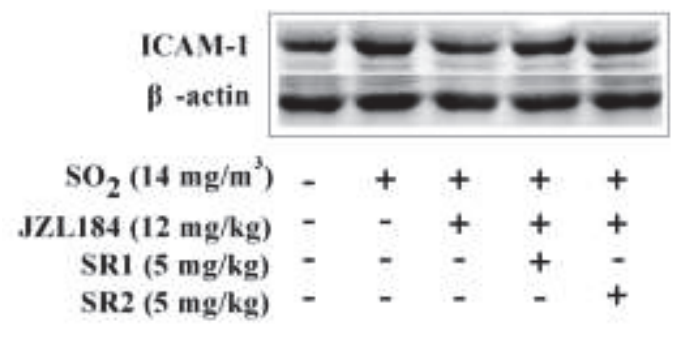

D1

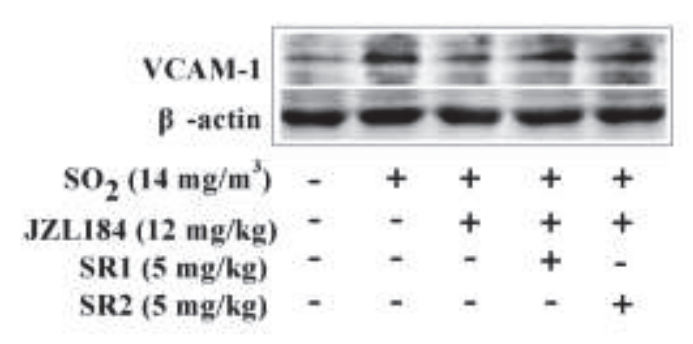

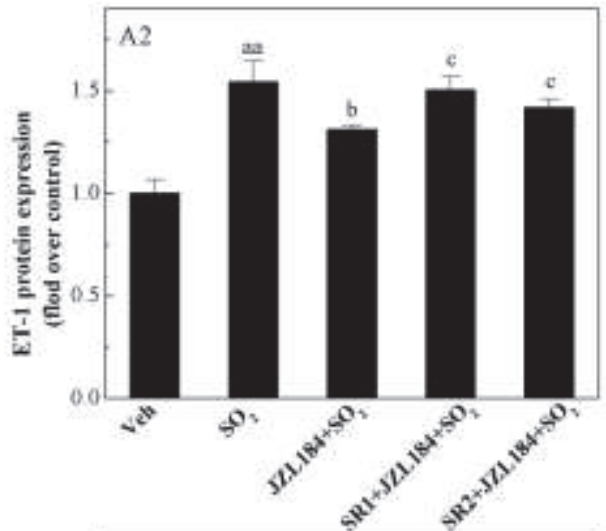
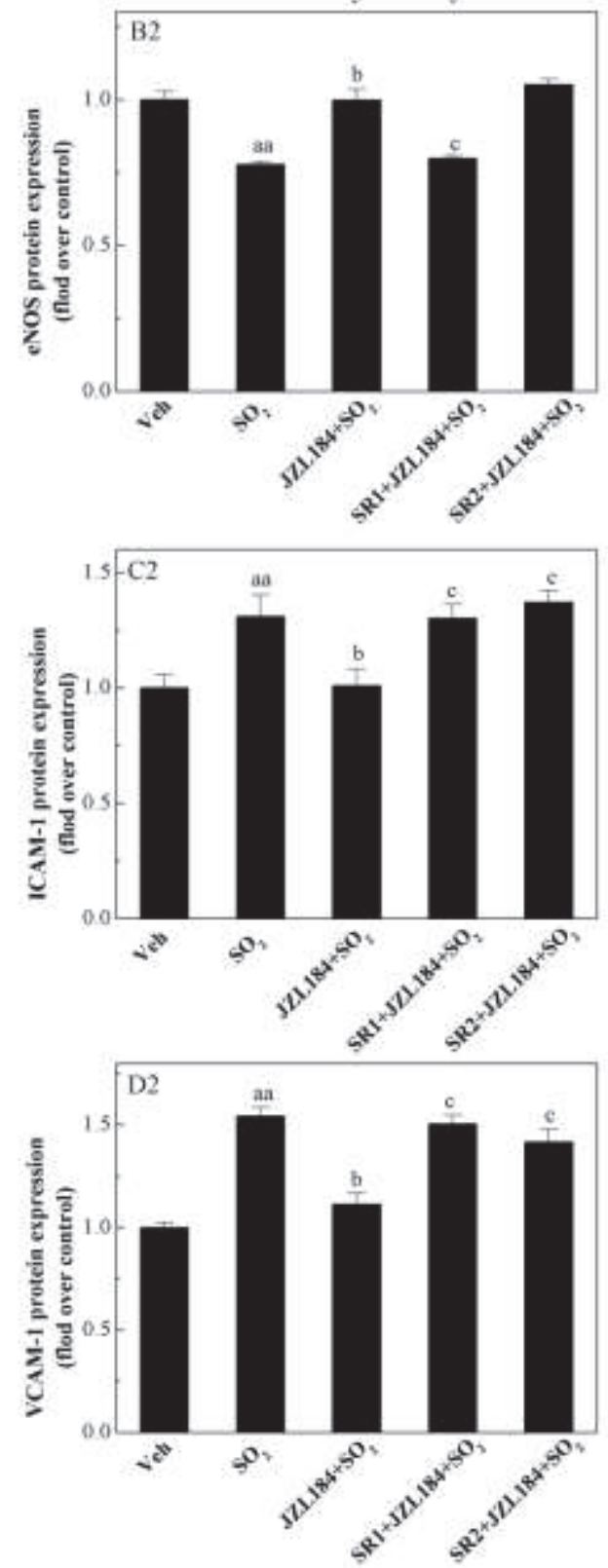

Fig. 5 Endogenous 2-AG abolishes ET-1, VCAM-1 and ICAM-1 elevation and eNOS attenuation by $\mathrm{CB} 1 / 2$ receptors. (A1) Immunoblot analysis of ET-1 in cortex of mice 
exposed to $\mathrm{SO}_{2}$ in the absent or present of JZL184, SR-1+JZL184 and SR-2+JZL184; (A2) Quantifications of ET-1 expression under different conditions ( $n=8)$; (B1) Immunoblot analysis of eNOS in cortex of mice exposed to $\mathrm{SO}_{2}$ in the absent or present of JZL184, SR-1+JZL184 and SR-2+JZL184. (B2) Quantifications of eNOS expression under different conditions ( $n=8)$. (C1) Immunoblot analysis of ICAM-1 in the cortex of mice exposed to $\mathrm{SO}_{2}$ in the absent or present of JZL184, SR-1+JZL184 and SR-2+JZL184. (C2) Quantifications of ICAM-1 expression under different conditions $(n=8)$. (D1) Immunoblot analysis of VCAM-1 in the cortex of mice exposed to $\mathrm{SO}_{2}$ in the absent or present of JZL184, SR-1+JZL184 and SR-2+JZL184. (D2) Quantifications of VCAM-1 expression under different conditions $(n=8)$. Abbreviations: +, with; -, without. ${ }^{\text {aa }} p<0.01$ compared with vehicle controls; ${ }^{\text {b }} p<0.05$ compared with $\mathrm{SO}_{2}$ group; ${ }^{c} p<0.05$ compared with $\mathrm{SO}_{2}$ group in the presence of JZL184. 